Astromony Letters, Vol. 32, No. 1, 2006

\title{
OUTER PSEUDORING IN THE GALAXY
}

\author{
A.M.Mel'nik \\ anna@sai.msu.ru \\ http://lnfm1.sai.msu.ru/ anna \\ Sternberg Astronomical Institute, Moscow, Russia \\ to be published in Astronomy Letters, 2006 \\ (C)2006 Pleiades Publishing, Inc.
}

\begin{abstract}
The kinematical features of the Sagittarius $(R=5.7 \mathrm{kpc})$, Carina $(R=6.5 \mathrm{kpc})$, Cygnus $(R=6.8 \mathrm{kpc})$, and Perseus $(R=8.2 \mathrm{kpc})$ arm fragments suggest the existence of two spiral patterns rotating at different angular velocities in the Galaxy. The inner spiral pattern represented by the Sagittarius arm rotates at the angular velocity of the bar, $\Omega_{\mathrm{b}}=60 \pm 5 \mathrm{~km} \mathrm{~s}^{-1} \mathrm{kpc}^{-1}$. The outer spiral pattern, which consists of the Carina, Cygnus and Perseus arms, rotates at a smaller angular velocity, $\Omega_{\mathrm{s}}=12-22 \mathrm{~km} \mathrm{~s}^{-1} \mathrm{kpc}^{-1}$. The existence of the outer slow tightly wound spiral pattern and the inner fast spiral pattern can be explained in terms of the results of numerical simulations of the dynamics of the outer pseudoring. The OLR of the bar must be located between the Sagittarius and Carina arms. The Cygnus arm appears as a connecting link between the fast and slow spiral patterns.

Key words: the Galaxy, spiral structure, kinematics and dynamics, resonances.
\end{abstract}




\section{INTRODUCTION}

The kinematics of gas in the central region, infrared photometry, star counts, and other modern tests prove the presence of the bar in the Galaxy. Elliptical orbits near the Galactic center allow many peculiarities of gas kinematics including the co-called $3 \mathrm{kpc}$ expanding arm to be explained (Peters, 1975; Liszt, Burton, 1980). There is extensive evidence suggesting that the major axis of the bar is oriented in the direction of $\theta_{\mathrm{b}}=15-45^{\circ}$ so that the tip of the bar nearest to the Sun lies in the first quadrant. However, observational estimates of the angular velocity of the bar and its length are rather uncertain (see Kuijken, 1996; Gerhard, 1996 and references therein).

In this paper we analyze the kinematics and distribution of OB-associations located within $3 \mathrm{kpc}$ of the Sun. A model of the Galaxy with an outer pseudoring made up of tightly wound spiral arms can explain the unusually small interarm distance $\lambda=2 \mathrm{kpc}$ between the Carina and Perseus arm fragments (Mel'nik et. al., 2001). The kinematics of young stars is clearly indicative of slow rotation of the spiral pattern represented by the Carina, Cygnus, and Perseus arm fragments, which is in a good agreement with numerical simulations of the dynamics of the outer pseudoring (Rautiainen and Salo, 1999, 2000). The galactic model with fast and slow rotating spiral patterns at different Galactocentric distances allows us to explain the fact that the kinematics of young stars in the Carina, Cygnus, and Perseus arms differs from that in the Sagittarius arm.

\section{NUMERICAL SIMULATIONS OF OUTER PSEUDORINGS AND CONFRONTING MODELS AND OBSERVATIONS}

Schwarz (1981) was the first to show that outer rings result from the evolution of barred galaxies. His numerical simulations showed two tightly wound spiral arms making up a pseudoring to develop in the galactic periphery after $\sim 10$ bar revolutions. The location of the outer pseudoring corresponds to the outer Lindblad resonance (OLR) of the bar. Schwarz (1981) found cloud-cloud collisions to play a crucial role in the formation of the outer pseudoring. Shocks in spiral arms emerging from the bar produce slow flow of gas clouds $\left(V_{R} \leq 1 \mathrm{~km} \mathrm{~s}^{-1}\right)$ towards the OLR and conduce to the gas accumulation in the OLR region. The time scale of this process is $10^{9}$ yeas. It determines the time needed for the galaxy to form an outer pseudoring. Schwarz discovered two types of the outer pseudorings - aligned with the bar and perpendicular to it. He associated them with two types of periodic orbits near the OLR. Periodic orbits between the corotation radius (CR) 
and the OLR are perpendicular to the bar, whereas orbits outside the OLR are aligned with the bar.

Buta (1986, 1995) discovered and studied more than 500 galaxies with outer rings or pseudorings (broken rings), of which $90 \%$ proved to be barred. Buta and Crocker (1991) found galaxies with well-defined characteristic features of each pseudoring type, which can be considered as prototypes. Type $R_{1}^{\prime}$ pseudorings are made up of spiral arms, which wind $\sim 180^{\circ}$ with respect to the ends of the bar. These pseudorings are aligned perpendicular to the bar and always lie inside the OLR of the bar. Type $R_{2}^{\prime}$ pseudorings are made up of spiral arms, which wind $\sim 270^{\circ}$ with respect to the ends of the bar. They are aligned with the bar and lie mostly outside the OLR. Figure 1 shows a schematic view of a typical galaxy with an $R_{2}^{\prime}$ pseudoring from the paper by Buta and Crocker (1991).

The simulation of ring galaxies carried out by Byrd et al. (1994) shows that first a type $R_{1}^{\prime}$ pseudoring appears and then it transforms into a type $R_{2}^{\prime}$ pseudoring. Type $R_{2}^{\prime}$ rings are, on average, located father away from the galactic center than type $R_{1}^{\prime}$ pseudorings, and therefore this sequence of pseudoring formation further emphasizes the important role that gas coming from the central region plays in the formation of outer rings.

What is the angular rotation velocity, $\Omega_{\mathrm{p}}$, of the tightly wound spiral pattern that forms the pseudoring? The above authors implied that the bar and the outer pseudoring rotate at the same angular velocity.

Another direction in modeling barred galaxies led to the discovery in galactic discs of several patterns rotating at different angular velocities (Sellwood, 1985; Sellwood and Sparke, 1988; and other papers). The slowly rotating spiral pattern was found at some distance from the galactic center. Tagger et al (1987) and Sygnet et al. (1988) developed a mechanism of nonlinear coupling between the bar and slowly rotating spiral pattern. They show that the bar and slow spiral pattern can exchange energy and angular momentum if the $\mathrm{CR}$ of the bar coincides with the inner Lindblad resonance (ILR) of the slow spiral mode.

Rautiainen and Salo (1999) were the first to find that the outer pseudoring may comprise spiral arms rotating slower than the bar. Numerical simulations show that in some cases a galaxy may possesses both inner and outer spiral arms. Inner arms rotate at the angular velocity of the bar, whereas outer tightly wound arms rotate at a lower velocity. The slowly rotating outer pseudoring is usually located at the outer edge of the OLR of the bar. Rautiainen and Salo (1999) also pointed out possible non-linear coupling of the two spiral modes so that the OLR of the inner spiral pattern coincides with the ILR of the outer spiral pattern. And this mechanism 
must differ from that suggested by Tagger (1987). Rautiainen and Salo (2000) performed $N$-body simulations of the evolution of a barred galaxy that showed the outer pseudoring to be usually located between the OLR of the bar and the CR of the slow spiral mode. Another interesting result of the above authors was the discovery of cyclic variations in the pseudoring morphology. First, a type $R_{1}^{\prime}$ pseudoring forms, then it acquires the features of a type $R_{2}^{\prime}$ pseudoring, then again those of a type $R_{1}^{\prime}$ pseudoring, and so on.

\section{UNUSUAL SAGITTARIUS ARM}

In our Galaxy young clusters and OB-associations located within $3 \mathrm{kpc}$ of the Sun concentrate toward several regions of intense star formation, which are traditionally associated with the Sagittarius-Carina, Cygnus-Orion, and Perseus spiral arms (Morgan et al., 1952; Becker, 1964; Humphreys, 1979 and other papers). However, the kinematics of young objects in the Carina, Cygnus, and Perseus complexes differs from that of young objects in the Orion and Sagittarius regions ( see Mel'nik et al., 2001 for details).

Fig. 2 shows regions of intense star formation within $3 \mathrm{kpc}$ of the Sun and the radial component $V_{R}$ of the average residual velocity of young stars in these regions. We computed the components of the stellar residual velocity along the Galactic radius-vector $V_{R}$ and in the azimuthal direction $V_{\theta}$ as differences of observed velocities and velocities due to the circular rotation law and solar motion relative to the centroid of OB associations projected onto each direction. We adopted the parameters of the circular Galactic rotation law and the components of solar motion from Mel'nik et al. (2001). We set the solar Galactocentric distance equal $R_{0}=7.1 \mathrm{kpc}$ (Dambis et al, 1995; Glushkova et al, 1998).

The radial velocity $V_{R}$ in the Carina, Cygnus, and Perseus complexes is directed toward the Galactic center whereas those in the Orion and Sagittarius regions $V_{R}$ are directed away from the Galactic center. The locations of the Carina, Cygnus, and Perseus spiral arms correspond to the extreme negative values of radial velocities $V_{R}$, i.e. to the maximum velocities toward the Galactic center (Fig. 2).

Simulations of the behavior of gaseous clouds in a perturbed potential performed by Roberts and Hausman (1984) showed that in trailing spiral arms located inside the $\mathrm{CR}$ the radial and azimuthal velocity components must reach their extreme negative values at the shock front. This is just the picture observed in the Cygnus $(R=6.8 \mathrm{kpc})$ and Perseus $(R=8.2 \mathrm{kpc})$ arms and it unambiguously indicates that these spiral arms are trailing 
and located inside the CR. We find no statistically significant variations in the azimuthal residual velocity $V_{\theta}$ across the Carina arm $(R=6.5 \mathrm{kpc})$. However, the Carina arm is closer to the Galactic center than the Cygnus and Perseus arms and therefore it must also lie inside the CR (Fig. 2). Hence all three fragments of the Carina, Cygnus, and Perseus arms are parts of a slowly rotating spiral pattern. The requirement that the Perseus arm is located inside the $\mathrm{CR}$ constrains the angular velocity of this pattern to $\Omega_{\mathrm{p}}<25 \mathrm{~km} \mathrm{~s}^{-1} \mathrm{kpc}^{-1}$ (see Mel'nik, 2003 for details).

The two regions of intense star formation in Orion and Sagittarius proved to be located in the interarm space. What triggers star formation in these regions? The Orion region is probably a spur of the Cygnus arm, because it is located at the outer edge of the arm. This is consistent with the conclusions of the studies of spurs by Weaver (1970) and Elmegreen (1980).

Intense star formation in the Sagittarius region is rather difficult to explain. This region contains two rich OB-associations (Sgr OB1 and Ser OB1) and has a size of $\sim 500 \mathrm{pc}$ in the azimuthal direction. We see no other spiral arm whose spur could form the complex of young objects in Sagittarius. Although extinction is extremely strong $\left(A_{V}=3^{m}\right)$ at heliocentric distances $1-2 \mathrm{kpc}$ in the direction $\left(l=28^{\circ}, b=-1^{\circ}\right)$, it remains rather moderate $\left(A_{V}<1^{m}\right)$ in the direction $\left(l=8^{\circ}, b=0^{\circ}\right)$ out to $r=2.5 \mathrm{kpc}$ and would not prevent the discovery of a "true" spiral arm (Neckel and Klare, 1980; Efremov and Sitnik, 1988). There is probably indeed no "true" spiral arm behind the Sagittarius region.

My principal assumption is that the star-forming region in Sagittarius is a fragment of a trailing spiral arm. It has been traditionally interpreted just in this way, or, to be more precise, as a constituent part of the SagittariusCarina arm. However, because of their kinematic differences the Sagittarius and Carina arms cannot be considered to be fragments of the same spiral pattern. The abrupt increase of extinction (from 2 to $4^{m}$ ) at heliocentric distances $2-3 \mathrm{kpc}$ in the field centered at $\left(l=18^{\circ}, b=0^{\circ}\right.$ ) (Neckel and Klare, 1980) also suggests that the Sagittarius region is a trailing spiral arm. Once adopted, this assumption immediately implies that the Sagittarius arm is a fragment of another spiral pattern, which rotates at a higher $\Omega_{\mathrm{p}}$, in contrast to the Carina, Cygnus and Perseus arms, which represent a slow spiral pattern.

Indeed the fact that the radial velocity $V_{R}$ of young stars in the Sagittarius arm is directed away from the Galactic center $\left(V_{R}=+10 \mathrm{~km} \mathrm{~s}^{-1}\right)$ indicates that this arm is located between the CR and OLR of its spiral pattern. Analytical calculations of stellar motions in tightly wound trailing spiral arms show that the direction of the radial component of velocity 
perturbation in the arm depends on the position of the arm with respect to the CR: it is directed toward and away from the Galactic center inside and outside the CR, respectively (Lin et al., 1969). In this paper we assume all spiral arms to be trailing.

In a spiral density wave the deviation of the velocities of young objects from circular rotation is due to their coherent motions along epicycles and the crowding of the orbits in the spiral arm corresponds to a certain phase of epicyclic motion. As long as the density wave exists, the crowding of the orbits in the stellar and gaseous subsystems of the disk must correspond to about the same phase of epicyclic motion and therefore the allowance for collisions does not alter the conclusion that the Sagittarius arm is located outside the CR.

The position of the Sagittarius arm between the CR and OLR means that the CR of this spiral pattern is closer to the Galactic center than the Sagittarius region $\left(R_{R C}<5.7 \mathrm{kpc}\right)$. Hence the angular velocity of the rotation of this spiral pattern must be greater than the angular velocity of Galactic rotation at the distance of the Sagittarius arm, $\Omega_{\mathrm{p}}>38 \mathrm{~km} \mathrm{~s}^{-1}$ $\mathrm{kpc}^{-1}$.

Thus there are two spiral patterns in the Galaxy, which rotate at different angular velocities. The spiral pattern that is closer to the Galactic center rotates faster $\left(\Omega_{\mathrm{p}}>38 \mathrm{~km} \mathrm{~s}^{-1} \mathrm{kpc}^{-1}\right)$ than the spiral pattern located farther from the Galactic center $\left(\Omega_{\mathrm{p}}<25 \mathrm{~km} \mathrm{~s}^{-1} \mathrm{kpc}^{-1}\right)$.

\section{IDENTIFICATION OF ARM FRAGMENTS WITH ELEMENTS OF THE PSEUDORING}

The fact that the Galactic radius-vector drawn at angles $\theta=10-15^{\circ}$ crosses two spiral arms - the Cygnus $(R=6.8 \mathrm{kpc})$ and Perseus $(R=8.2 \mathrm{kpc})$ fragments - suggests that the Galaxy possesses a tightly wound and not a multi-armed spiral pattern (Fig. 2). Fast periodic density variations along the radius are virtually impossible in the multi-armed spiral pattern because of the large pitch angle of spiral arms.

Owing to the $\sim 270^{\circ}$ winding of the spiral arms with respect to the bar, type $R_{2}^{\prime}$ pseudorings have regions where the same galactic radius-vector crosses two tightly wound spiral arms (Fig. 1). These regions lie at smaller angles $\theta$ relative to the direction of elongation of the bar, i.e., they go slightly behind the bar (Buta, Crocker, 1991). On the average, the direction $\theta=$ $10-15^{\circ}$ in the Galaxy toward which the double spiral arms are observed somewhat lags behind the direction of elongation of the bar, $\theta_{\mathrm{b}}=15-45^{\circ}$, 
and this fact agrees well with the type $R_{2}^{\prime}$ pseudoring model.

The termination of the Perseus arm in the III quadrant provides additional evidence for the presence of a type $R_{2}^{\prime}$ pseudoring in the Galaxy. The $R_{2}^{\prime}$ pseudoring must be stretched along the bar. Hence the tightly wound spiral arms forming the pseudoring must terminate in the direction of bar elongation. In the solar neighborhood the Perseus arm is the most distant one from the Galactic Center. The termination of the Perseus arm at Per OB1 association $\left(\theta=9^{\circ}\right)$ agrees rather well with the direction of the bar elongation, $\theta_{\mathrm{b}}=15-45^{\circ}$.

Thus the morphology and kinematics of the Galaxy suggest the presence of a type $R_{2}^{\prime}$ pseudoring. Numerical simulations indicate that the type $R_{2}^{\prime}$ pseudoring must lie mostly outside the OLR of the bar. For numerical simulations to be consistent with the kinematic features of observed spiral arms, one must assume that the OLR of the bar lies between the Sagittarius and Carina arms (Fig. 2). The Carina, Cygnus, and Perseus arms, which represent the slow spiral pattern, would then be located outside the OLR, whereas the Sagittarius arm, which represents the fast spiral pattern, would be located inside the OLR. Such a picture is consistent with the presence in the Galaxy of inner and outer spiral arms. Inner spiral arms must lie between the CR and OLR of the bar and rotate at the angular velocity of the bar, $\Omega_{\mathrm{b}}$. Outer, tightly wound spiral arms must lie at the outer edge of the OLR and rotate slower than the bar. (Rautiainen and Salo, 1999, 2000).

\section{FAST ROTATING SPIRAL PATTERN}

The localization of the OLR of the bar between the fragments of the Sagittarius $(R=5.7 \mathrm{kpc})$ and Carina $(R=6.5 \mathrm{kpc})$ arms allows its angular rotation velocity $\Omega_{\mathrm{b}}$ to be rather accurately determined:

$$
\Omega_{\mathrm{b}}=\Omega\left(R_{\mathrm{OLR}}\right)+\kappa\left(R_{\mathrm{OLR}}\right) / 2
$$

where $\Omega\left(R_{O L R}\right)$ is the average angular velocity of disk rotation and $\kappa\left(R_{O L R}\right)$ is the epicyclic frequency at the distance of the OLR. We use the parameters of the circular law of Galactic rotation from Mel'nik et al. (2001) to obtain $\Omega_{\mathrm{p}}=60 \pm 5 \mathrm{~km} \mathrm{~s}^{-1} \mathrm{kpc}^{-1}$. The error in $\Omega_{\mathrm{b}}$ is mostly determined by the accuracy of the localization of the OLR. In this case no question arises about the number of spiral arms. Both the bar and associated spiral pattern must have the mode $\mathrm{m}=2$.

The CR of the bar rotating at angular velocity of $\Omega_{\mathrm{b}}=60 \pm 5 \mathrm{~km}$ $\mathrm{s}^{-1} \mathrm{kpc}^{-1}$ must lie at the distance of $R_{R C}=3.3 \pm 0.5 \mathrm{kpc}$. Weiner and 
Sellwood (1999) obtained similar locations for the CR and OLR of the bar $\left(R_{\mathrm{CR}}=3.6 \mathrm{kpc}\right.$ and $\left.R_{\mathrm{OLR}} \approx R_{0}\right)$ by analyzing the $(l-V)$ diagrams of $\mathrm{HI}$ and $\mathrm{CO}$ distributions in the central region of the Galaxy.

Fig. 1 clearly shows the difference in the pitch angles of the spiral arms in the inner and outer galactic regions. The pitch angle of the spiral arms in the inner and outer regions are close to $90^{\circ}$ and $0^{\circ}$, respectively. The change in the pitch angle of spiral arms near the OLR of the bar was pointed out by Schwarz (1981), Combes and Gerin (1985), and other authors. Let us assume that the inner spiral arm begins at the $\mathrm{CR}$ of the bar at $(\mathrm{R}=3.3 \mathrm{kpc}$, $\left.\theta=25^{\circ}\right)$ and passes through the Sagittarius complex $\left(R=5.7 \mathrm{kpc}, \theta=3^{\circ}\right)$. The average pitch angle of inner spiral arms must then be equal to $i=55^{\circ}$.

\section{SLOW ROTATING SPIRAL PATTERN}

Let us now consider the slowly rotating spiral pattern, which is represented by the Carina, Cygnus, and Perseus arm fragments. In most of the cases pseudorings in galaxies consist of two tightly wound spiral arms (Buta, 1995). In the Galaxy such arms are probably represented by the Carina and Perseus arm fragments. For the two-armed model $\left(m_{s}=2\right)$ the pitch angle of the outer spiral arms must be equal to $i=5^{\circ}$ (Mel'nik et al., 2001).

The Carina $\operatorname{arm}(R=6.5 \mathrm{kpc})$ is likely to be located near the ILR of the slowly rotating spiral pattern. This is evidenced by the specific features of its kinematics and the lack of other arms of the slow spiral pattern at smaller $R$. The fast spiral pattern is already present at $R=5.7 \mathrm{kpc}$ and therefore the slow spiral pattern may extend inward only down to Galactocentric distances $R \approx 5.7 \mathrm{kpc}$ (Mel'nik et al., 2005).

The kinematic peculiarity of the Carina arm - namely the absence of significant cross-arm variations of the azimuthal velocity component - probably suggests that the arm has degenerated into a ring. It is very difficult to explain the presence of cross-arm gradient of radial velocity combined with the absence of cross-arm gradient of azimuthal velocity. In a spiral density wave both $V_{R}$ and $V_{\theta}$ velocity components must necessarily exhibit cross-arm variations. Both velocity gradients are due to the same cause: the change of the direction of elongation of orbits in the disk plane with increasing Galactocentric distance $R$.

The observed gradient of the radial velocity $V_{R}$ across the Carina arm may be due to the contraction of orbits located near the ILR. In the ILR region gaseous clouds must effectively lose angular momentum and acquire radial velocity directed toward the Galactic center, while organized motions in the azimuthal direction may be absent. 
Let us assume that the ILR of the slow spiral mode is located at the Galactocentric distance of the Carina arm $R=6.5 \mathrm{kpc}$. If we knew the number of spiral arms $m_{s}$ in the outer spiral pattern, we could immediately compute the corresponding pattern speed $\Omega_{\mathrm{s}}$ :

$$
\Omega_{\mathrm{s}}=\Omega\left(R_{\mathrm{ILR}}\right)-\kappa\left(R_{\mathrm{ILR}}\right) / m_{s}
$$

where $\Omega\left(R_{\mathrm{ILR}}\right)$ and $\kappa\left(R_{\mathrm{ILR}}\right)$ are the average angular velocity of disk rotation and the epicyclic frequency, respectively, calculated at the distance of the ILR.

In the Galaxy we are dealing with a small number of tightly wound spiral arms. However, we cannot be sure that the Galaxy has 2 and not 4 arms. The $m_{s}=2$ and $m_{s}=4$ models imply the pattern speeds of $\Omega_{\mathrm{s}}=12 \pm 2$ and $\Omega_{\mathrm{s}}=22 \pm 2 \mathrm{~km} \mathrm{~s}^{-1} \mathrm{kpc}^{-1}$, respectively. Both values obey the inequality $\Omega_{\mathrm{s}}<25 \mathrm{~km} \mathrm{~s}^{-1} \mathrm{kpc}^{-1}$, which expresses the requirement that the Perseus arm is located inside the CR. Note that in the two-armed model $\left(m_{s}=2\right)$ the $\mathrm{CR}$ of the slow spiral mode is located at the Galactocentric distance of $R_{\mathrm{RC}} \approx 15-17 \mathrm{kpc}$, whereas in the four-armed model $\left(m_{s}=4\right)$ it is located at $R_{\mathrm{RC}} \approx 10 \mathrm{kpc}$. It is rather difficult to choose one of the two models, but in any case the angular velocity of rotation of the slow spiral pattern must lie in the interval of $\Omega_{\mathrm{s}}=12-22 \mathrm{~km} \mathrm{~s}^{-1} \mathrm{kpc}^{-1}$.

Fig. 3 shows a schematic view of the spiral structure of the Galaxy under the assumption of two-armed symmetry. We set the angle between the major axis of the bar and the solar direction equal to $\theta_{\mathrm{b}}=25^{\circ}$ and the axial ratio of the bar to be equal to 3:1 (Gerhard, 1996; Weiner, Sellwood, 1999). The pith angles of the inner and outer spirals are equal to $i=55^{\circ}$ and $i=5^{\circ}$, respectively. The dashed line shows the position of the OLR of the bar. The locations of the star-gas complexes within $3 \mathrm{kpc}$ of the Sun are carried over from Fig. 2.

\section{THE CYGNUS ARM AS A CONNECTING LINK BETWEEN THE} FAST AND SLOW SPIRAL PATTERNS

Thus the Galaxy possesses a fast $\left(\Omega_{\mathrm{p}}=60 \pm 5 \mathrm{~km} \mathrm{~s}^{-1} \mathrm{kpc}^{-1}\right)$ and a slow $\left(\Omega_{\mathrm{s}}=12-22 \mathrm{~km} \mathrm{~s}^{-1} \mathrm{kpc}^{-1}\right)$ spiral patterns. How to explain such a good coupling of the ends of these two patterns? Complexes of young objects in Sagittarius $\left(\theta=3^{\circ}\right)$ and Carina $\left(\theta=-15^{\circ}\right)$ are $\Delta \theta=18^{\circ}$, or only $5 \%$ of the circumference, apart. It is this accurate coupling that gave rise to the emergence of the notion of the Sagittarius-Carina arm, although different 
parts of this arm move at an angular velocity of $\Delta \Omega=40-50 \mathrm{~km} \mathrm{~s}^{-1} \mathrm{kpc}^{-1}$ relative to each other.

We can answer this question if we assume that both spiral patterns are periodically deformed and adjust to each other. The Cygnus arm is very much like a connecting link between the fast and slow spiral patterns. In Fig. 3 the Cygnus arm and the arm fragment that is symmetric to it are located at the places where the inner and outer spirals join each other forming specific "cap-peaks". These "cap-peaks" make outer spirals appear to lead the inner spirals in Galactocentric angle. Sellwood and Spark (1988) found similar connecting links between the fast rotating bar and slow rotating spiral pattern.

It is interesting that the ILR of the slow spiral pattern $(R \approx 6.5 \mathrm{kpc})$ is located near the OLR of the fast spiral pattern $(R \approx 6.1 \mathrm{kpc})$. Rautiainen and Salo $(1999,2000)$ pointed out the close location of the resonances, the OLR and ILR, of the two spiral patterns in numerical simulations. Gaseous clouds, which may accumulate in the narrow ring between the OLR of the inner and the ILR of the outer spiral mode must play important role in the mechanism of coupling of resonances OLR-ILR. The fast spiral mode forms a flow of gaseous clouds from the center toward the periphery (Schwarz, 1981), whereas the slow spiral mode produces a flow in the opposite direction, from the periphery toward the center. Possibly two spiral modes periodically exchange gaseous clouds and amplify each other.

\section{CONCLUSIONS}

The kinematic features of the Sagittarius, Carina, Cygnus, and Perseus arms suggest that the Galaxy possesses two spiral patterns rotating at different angular velocities. The Sagittarius arm is a fragment of a spiral pattern that rotates at a higher angular velocity than the pattern represented by the Carina, Cygnus, and Perseus arms. The presence of a slow outer tightly wound spiral pattern and a fast inner spiral pattern can be explained using numerical simulations of the dynamics of outer pseudorings (Rautiainen and Salo, 1999, 2000). The OLR of the bar must then be located between the Sagittarius and Carina arms. In this case the Carina, Cygnus, and Perseus arms, which represent the slow spiral pattern, must be located outside the OLR, whereas the Sagittarius arm, which represents the fast spiral pattern, must lie inside the OLR of the bar.

The localization of the OLR of the bar between the Sagittarius and Carina arms allows us to determine the angular velocity of bar rotation $\Omega_{\mathrm{b}}=60 \pm 5 \mathrm{~km} \mathrm{~s}^{-1} \mathrm{kpc}^{-1}$, and the radius of bar coratation $R_{\mathrm{RC}}=3.3 \pm 0.5$ 
kpc. The angular velocity of the rotation of the slow spiral pattern must lie in the interval $\Omega_{\mathrm{s}}=12-22 \mathrm{~km} \mathrm{~s}^{-1} \mathrm{kpc}^{-1}$.

\section{ACKNOWLEDGMENTS}

I am grateful to A. V. Zasov, I. I. Pasha, Yu. N. Efremov, and A. S. Rastorguev for interesting discussion and useful remarks. This work was supported by the Russian Foundation for Basic Research (projects nos. 03-02-16288 and 04-02-16689), the Council for the Program of Support for Leading Scientific Schools (project no. NSh.389.2003.2), and the State Science and Technology Astronomy Program.

\section{REFERENCES}

1. W. Becker, The Galaxy and the Magellanic Clouds, IAU Symp.20, Ed. F. J. Kerr (Canberra: Austral. Acad. Sci., 1964), p.16

2. R. Buta, Astrophys. J. Suppl. Ser. 61, 609 (1986).

3. R. Buta, Astrophys. J. Suppl. Ser. 96, 39 (1995).

4. R. Buta and D. A. Crocker, Astron. J. 102, 1715 (1991).

5. G. Byrd, P. Rautiainen, H. Salo, R. Buta and D. A. Crocker, Astron. J. 108, 476 (1994).

6. F. Combes and M. Gerin, Astron. Astrophys. 150, 327 (1985).

7. A. K. Dambis, A. M. Mel'nik, and A. S. Rastorguev, Astron. Lett. 21, 291 (1995).

8. Yu.N. Efremov and T.G. Sitnik, Astron. Lett. 14, 347 (1988).

9. D. M. Elmegreen, Astrophys. J. 242, 528 (1980).

10. O. E. Gerhard, Unsolved Problems of the Milky Way, IAU Symp. 169, Ed. L. Blitz, P. Teuben (Dordrecht: Kluwer, 1996), p.79.

11. E. V. Glushkova, A. K. Dambis, A. M. Mel'nik, and A. S. Rastorguev, Astron. Astrophys. 329, 514 (1998).

12. R. M. Humphreys, The Large Scale Characteristics of the Galaxy, IAU Symp. 84, Ed. W. B. Burton (Dordrecht: D. Reidel Publ. Co., 1979), p.93. 
13. K. Kuijken, ASP Conference Ser. 91, 504, (1996).

14. C. C. Lin, C. Yuan, and F. H. Shu, Astrophys. J. 155, 721 (1969).

15. H. S. Liszt and W. B. Burton, Astrophys. J. 236, 779 (1980).

16. A. M. Mel'nik, Astron. Lett. 29, 304 (2003).

17. A. M. Mel'nik, Astron. Lett. 31, 80 (2005).

18. A. M. Mel'nik, A. K. Dambis, and A. S. Rastorguev, Astron. Lett. 27, 521 (2001).

19. W. W. Morgan, S. Sharpless, and D. Osterbrock, Astron. J. 57, 3 (1952).

20. Th. Neckel and G. Klare, Astron. Astrophys. Suppl. Ser. 42, 251 (1980).

21. W. L. Peters, Astrophys. J. 195, 617 (1975).

22. P. Rautiainen and H. Salo, Astron. Astrophys. 348, 737 (1999).

23. P. Rautiainen and H. Salo, Astron. Astrophys. 362, 465 (2000).

24. W. W. Roberts, and M. A. Hausman, Astrophys. J. 277, 744 (1984).

25. M. P. Schwarz, Astrophys. J. 247, 77 (1981).

26. J. A. Sellwood, Mon. Not. R. Astron. Soc. 217, 127 (1985).

27. J. A. Sellwood and L. S. Sparke, Mon. Not. R. Astron. Soc. 231, 25 (1988).

28. J. F. Sygnet, M. Tagger, E. Athanassoula, R. Pellat, Mon. Not. R. Astron. Soc. 232, 733 (1988).

29. M. Tagger, J. F. Sygnet, E. Athanassoula, R. Pellat, Astrophys. J. (Letters) 318, L43 (1987).

30. H. Weaver, Interstellar Gas Dynamics, IAU Symp. 39, Ed. H. Habing (Dordrecht: D. Reidel Publ. Co., 1970), p.22 .

31. B. J. Weiner and J. A. Sellwood, Astrophys. J. 524, 112 (1999). 
Astromony Letters, Vol. 32, No. 1, 2006

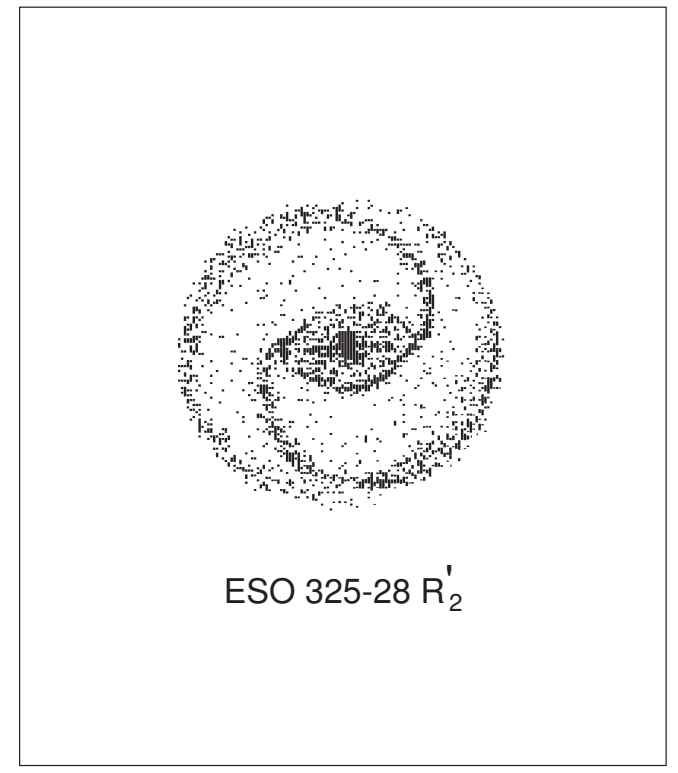

Figure 1: Image of a typical galaxy with pseudoring $R_{2}^{\prime}$ from Buta and Crocker (1991) (a mirror copy). 


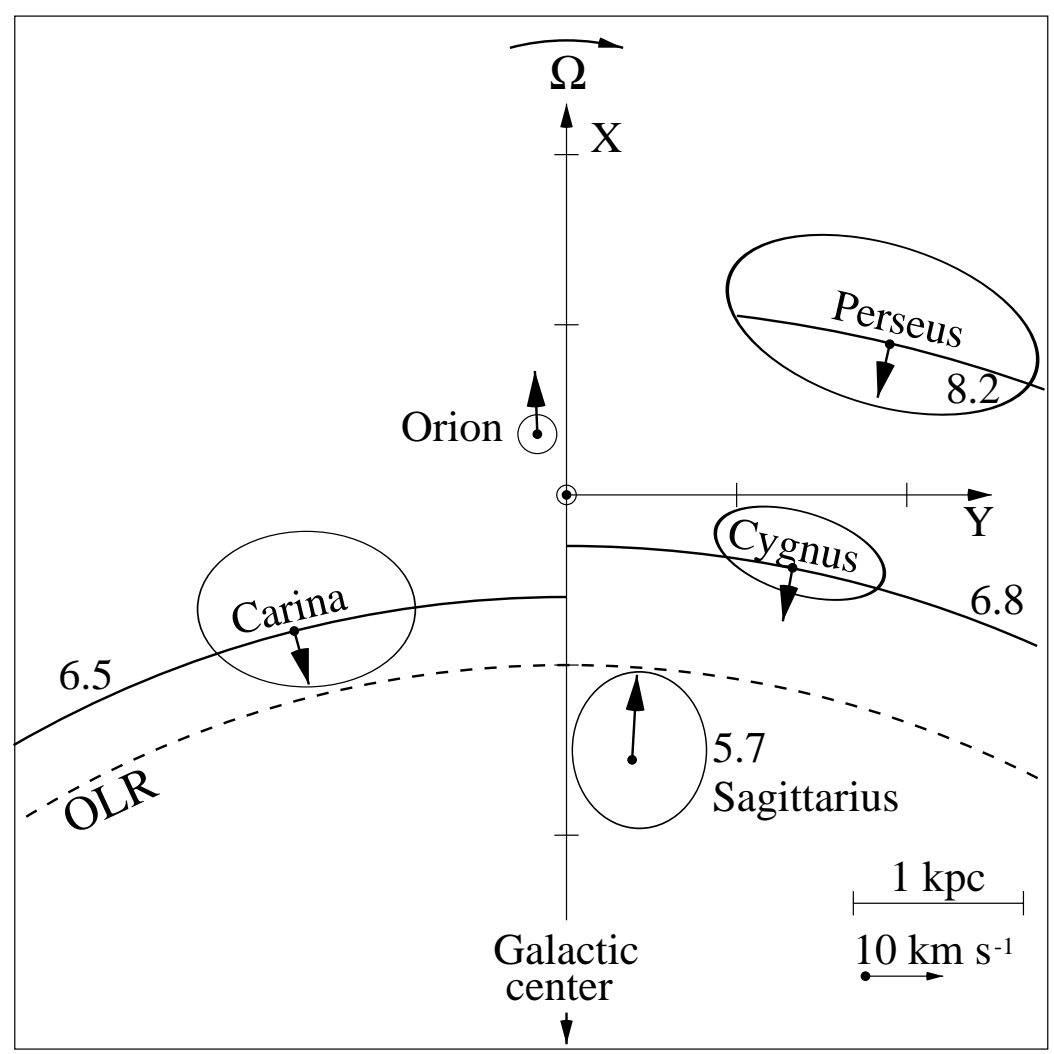

Figure 2: Regions of intense star formation in the Galaxy and their average radial velocity $V_{R}$. The locations of the Carina, Cygnus, and Perseus spiral arms correspond to the extreme negative values of radial velocity $V_{R}$. The dashed line shows the location of the OLR of the bar. The numbers indicate Galactocentric distances in kpc. 


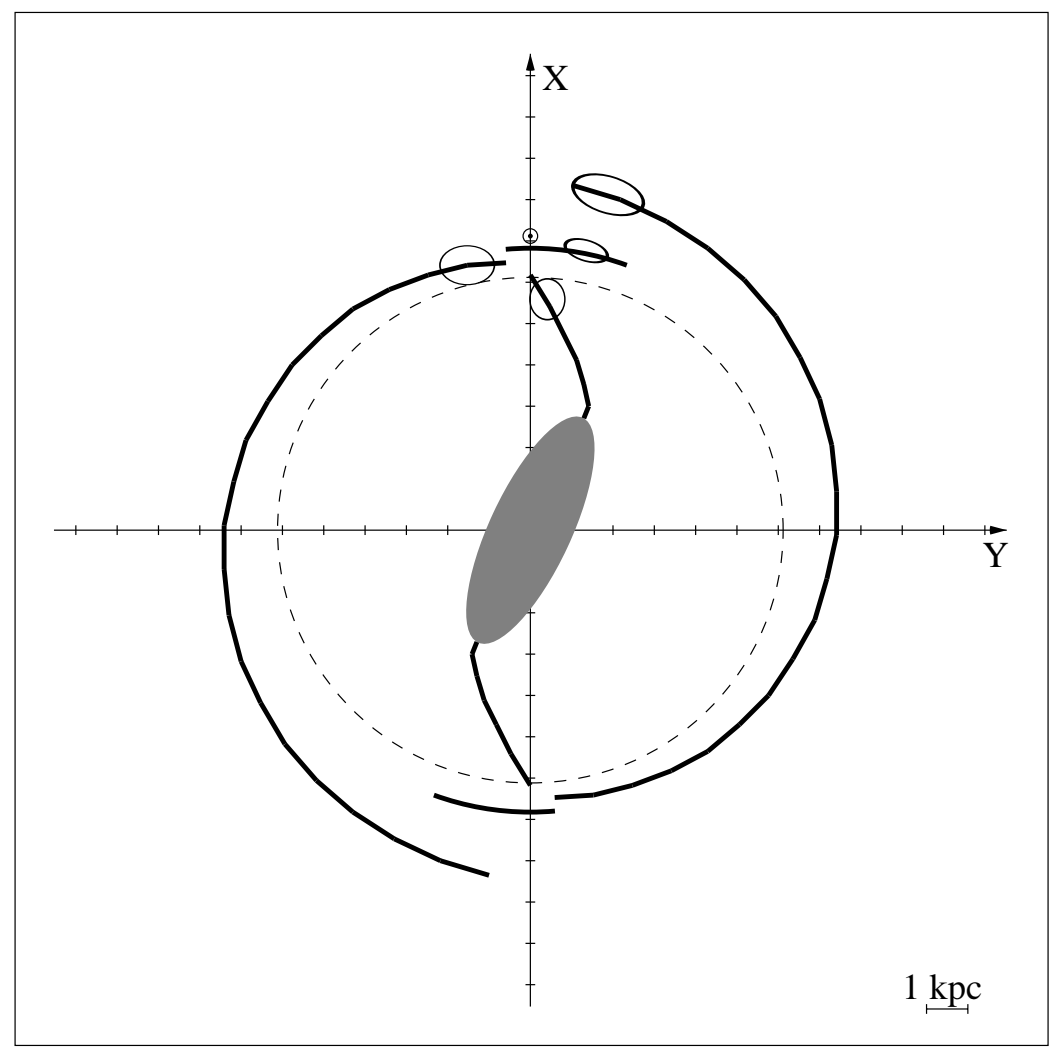

Figure 3: Schematic view of the spiral structure of the Galaxy. The pitch angles of the inner and outer spiral arms are equal to $i=55^{\circ}$ and $i=5^{\circ}$, respectively. The position of the Sun is indicated by the traditional sign. The positions of star-gas complexes within $3 \mathrm{kpc}$ are shown by ovals as in Fig. 2. The dashed line shows the location of the OLR of the bar. 